\title{
Importancia del módulo dinámico de rigidez del suelo en el análisis de los problemas de estabilidad por la acción de las ondas sísmicas
}

\author{
L. Zeevaert-Wiechers \\ División de Estudios de Posgrado, Facultad de Ingeniería, UNAM
}

(recibido: enero de 1999; aceptado: mayo de 2000)

Resumen

\begin{abstract}
El objetivo de este trabajo es el de motivar a los ingenieros interesados en mecánica y dinámica de suelos, al uso, aplicación y desarrollo de la sismo-geodinámica en la práctica profesional de la ingeniería sísmica, así como al estudio e investigación de los parámetros dinámicos del suelo. Para este fin, el autor presenta dos equipos que ha usado con éxito durante varias décadas.
\end{abstract}

Descriptores: sismo-geodinámica, parámetros, equipos

\begin{abstract}
The object of the present paper is to motivate engineers interested in soil mechanics and in soil dynamics to use, apply and develop seismic-geodynamics principles in the professional practice of seismic engineering, as well as to study and investigate dynamic soil parameters. For this purpose the author presents two types of equipment he has used with success during several decades.
\end{abstract}

Keywords: seismic-geodynamics; parameters; equipment.

\section{Prólogo}

En los últimos tiempos, se ha incrementado el interés por conocer con mayor precisión el efecto que causan las ondas sísmicas en los problemas relacionados con el subsuelo, las cimentaciones y obras de ingeniería civil en general; así también, en todas aquéllas regiones sujetas a sismos destructores.

El autor se ha dedicado durante varios años a tratar de entender racionalmente los problemas de la sismo-geodinámica y su aplicación práctica, tanto en México, como en el extranjero, esto en las zonas más afectadas por sismos ${ }^{1}$.

El objeto de los estudios realizados por el autor, en su mayoría publicados ${ }^{1}$, se han realizado con el deseo de interpretar la acción física de las ondas sísmicas y poder producir herramientas prácticas racionales para la profesión, las cuales puedan aplicarse con mayor grado de seguridad que los procedimientos empíricos. Así también, para fomentar el interés de la dinámica de suelos y la sismogeodinámica en los jóvenes interesados en el tema, creando en ellos una motivación que genere la continuidad en las investigaciones de esta apasionante disciplina tan necesaria para la profesión y la comunidad.

\section{Introducción}

En las últimas dos décadas, la ciencia de la ingeniería ha tratado de entender mejor los efectos que originan los sismos en el subsuelo, en las cimentaciones, así como en los edificios. De la misma forma, se han tratado de justificar y analizar por las leyes físicas de la naturaleza que rigen en el

\footnotetext{
${ }^{1}$ Lista de Publicaciones L.Z.W. DEPFI, UNAM.
} 
DOI: http://dx.doi.org/10.22201/fi.25940732e.2000.01n4.018

comportamiento geo-dinámico del subsuelo, dejando así, en forma gradual, los procedimientos empíricos que se han utilizado en el pasado y que todavía en ocasiones se hace uso de ellos.

Los ingenieros especializados en la mecánica y dinámica de suelos, conocen que el suelo en la naturaleza se presenta con múltiples características que dependen de su origen y formación en el ambiente de la región. Ejemplos palpables de esto lo constituyen los suelos residuales que cubren en gran parte la corteza terrestre, así como los suelos eólicos finos que se forman en climas áridos, lacustres y marinos, así como otros sedimentos de gran variedad como los aluviales y aún los de piemonte (Van Nostrand,1983).

Por lo anterior, se comprende que es imposible obtener propiedades cuantitativas por métodos empíricos de estadística que representen la totalidad de los suelos con diferentes características y propiedades físicas, y menos, pretender conocer con certidumbre los parámetros cuantitativos estáticos y dinámicos. De la misma manera, es difícil establecer reglas empíricas estadísticas para resolver en particular el problema de estabilidad que se presenta en cualquier suelo y sitio.

El suelo es altamente sensible a la acción dinámica, sus propiedades dependen de varios factores físicos y de las condiciones ambientales con que se encuentran en la naturaleza.

Las características físicas que más afectan a las propiedades mecánicas del suelo y que es necesario preservar al llevar a cabo muestreos así como durante el manejo del suelo en el laboratorio, es la estructura interna del suelo y los cambios en el grado de saturación, principalmente en los suelos arcillosos (Zeevaert, 1997).

En el laboratorio, las pruebas deberán efectuarse con el esfuerzo octaédrico, (Nadaí,1950) al que estaba sometido. el suelo en el campo y con la profundidad de donde se obtuvo la muestra, así como con el contenido de agua natural con que se encuentra en el campo.

El suelo es muy sensible a los factores antes mencionados, principalmente los que contienen minerales de arcilla con limo y arena.

Es evidente que las soluciones generales empíricas son prácticas y económicas, pero la experiencia de campo ha demostrado en múltiples ocasiones que su uso conduce a fallas que originan costos importantes de reparación, y en ocasiones pérdida total de las obras afectadas.

Lo anterior se puede reducir de manera efectiva si se llevaran a cabo previamente estudios físicos racionales del conocimiento dinámico del suelo en cuestión y de los parámetros que muestren en el campo el problema sismo-geodinámico que se presenta (Zeevaert,1997 y 1979). Es obvio que el costo de los análisis básicos es mayor si se compara con el uso de los métodos estadísticos empíricos; sin embargo, el costo de los estudios racionales es una fracción muy pequeña del costo de la obra a realizar.

Resulta incomprensible que el inversionista de una obra, aun de la magnitud que ésta sea, quiera omitir los estudios básicos que le brindarán mayor seguridad a su inversión.

Módulo de rigidez del suelo para analizar la acción de las ondas sísmicas

El módulo dinámico de rigidez del suelo es necesario para analizar técnicamente la acción de las ondas sísmicas en los siguientes problemas (Zeevaert, 1998):

1. Cálculo de la celeridad de las ondas (Zeevaert, 1993).

a) De cortante o equivolumétricas por la distorsión angular.

b) Irrotacionales, de compresión y dilatación.

c) Componente de la onda superficial de compresión y dilatación.

2. Períodos de vibración del suelo (Zeevaert, 1996).

3. Presiones, deformaciones unitarias y aceleraciones que se originan en el subsuelo en cada uno de los estratos que lo forman. Para el cálculo ver (Zeevaert, 1982 y 1996).

4. Presión sísmica en el agua de poro del suelo (Zeevaert, 1982).

5. Ángulo de fricción interna durante la acción sísmica (Zeevaert, 1997).

6. Capacidad de carga sísmica (Ídem).

7. Falla de la superficie del suelo por tracción de las ondas (Zeevaert, 1979).

8. Falla de la superficie del suelo por la compresión de las ondas (Ídem).

9. Profundidad de grietas sísmicas en la superficie del suelo (Ídem).

10. Reducción de la fricción positiva y negativa en pilotes (Zeevaert, 1995).

11. Reducción de la capacidad de carga en pilotes apoyados de punta (Zeevaert, 1997).

12. Cálculo de la flexo-compresión sísmica en pilotes (Zeevaert, 1996).

13. Estabilidad de las obras de tierra y cimentaciones (Nadaí, 1950).

14. Estabilidad de muros de retención, anclas y excavaciones en general (Zeevaert 1 ).

El análisis de los problemas antes citados se puede consultar en las referencias que se indican. Para resolver estos problemas es necesario el conocimiento de:

a) El perfil estratigráfico y las características índice de los suelos: Oquedad (e), contenido de agua (W), grado de 
saturación $(\mathrm{G} \%)$, presiones hidráulicas $(\mathrm{Pw})$, esfuerzos efectivos $(\sigma z)$, esfuerzos octaédricos $(\sigma c)$, presiones ejercidas por la onda sísmica $\left(\mathrm{Pxz}_{\mathrm{x}}\right)$, cohesión y ángulo de fricción interna para las condiciones estáticas y sísmicas.

b) Las presiones hidráulicas estáticas que se observan en el campo por medio de piezómetros instalados a profundidades previamente seleccionadas de acuerdo a la estratigrafía del sitio.

c) Muestreo inalterado del suelo.

d) Módulo dinámico de rigidez $(\mu)$, se investiga por medio del "Péndulo de torsión libre".

e) Celeridad de la onda sísmica de cortante en cada estrato del subsuelo:

$\mathrm{C}_{\mathrm{x} z}^{2}=\mu / \gamma$, donde $\gamma$ es la densidad unitaria del suelo.

f) Módulos de deformación unitaria: de respuesta vertical (Mez) y de compresión horizontal (Mxz); se pueden obtener con: "La cámara holandesa modificada". Luego: $\beta=\mathrm{Me} z / \mathrm{Mxz}$.

i) Perfil de los elementos sísmicos inducidos por la onda; presiones en el suelo, presión en el agua de poro, esfuerzos efectivos, deformaciones y aceleraciones con respecto a la asignada a la superficie del suelo, para todos los estratos que configuran el subsuelo y que caracterizan la investigación.

j) Cálculo de la presión sísmica en el agua de poro (Zeevaert, 1982).

k) Ángulo sísmico de fricción interna; $\phi$ sis.

1) Capacidad de carga sísmica.

Los módulos: $\mu$, Mez y Mxz son básicos para los análisis sísmicos de estabilidad y se obtienen de probetas de suelo inalterado, bajo las mismas condiciones en las que se encuentran en el campo, esto es, respetando el esfuerzo octaédrico y el grado de saturación.

Las herramientas para los métodos de análisis y cálculo de los problemas antes mencionados se pueden encontrar en las referencias que se citan.

\section{Módulo dinámico de rigidez (Zeevaert, 1982)}

Esta propiedad dinámica del suelo se investiga en el laboratorio con un equipo que pueda inducir en una probeta de suelo inalterado una vibración con períodos que representen aquellos que se espera tener en el campo por las ondas sísmicas.

Existen dos tipos de instrumentos confiables con los cuales se puede investigar en el laboratorio el módulo dinámico de rigidez:

1. La cámara de vibración forzada que fue originada por el profesor Frank Richart de la Universidad de Michigan.
En este instrumento se somete la probeta a una vibración forzada hasta que entre en resonancia. El uso de esta herramienta se puede consultar en las publicaciones de otros autores (Drnevich y Hardin) quienes han experimentado con ella (Hardin, et al., 1978 y Zeevaert 2).

2. El péndulo de torsión libre que fue introducido por el autor hace más de tres décadas en la Universidad Nacional Autónoma de México (Zeevaert, 1982).

La acción de este instrumento consiste en medir la vibración libre por la acción del esfuerzo cortante puro en una probeta sujeta a cierto estado de esfuerzo octaédrico y determinado grado de saturación de campo.

El péndulo de torsión libre ha sido mejorado por el profesor Van Impe W., en la Universidad Estatal de Ghent, en Bélgica y por el profesor Taylor P.W. en la Universidad de Auckland en New Zeland (Taylor, et al., 1973; Parton, 1972 y Van Impe, 1977).

En este trabajo, se presenta únicamente en forma somera el método de operación del péndulo de torsión libre, se hace hincapié en que las probetas que se utilicen deberán ser representativas e inalteradas del suelo por estudiar, lo cual se puede lograr cuando el suelo presenta suficiente consistencia natural que permita evitar que se altere su estructura interna al recabar muestras cúbicas, o bien, utilizando muestreadores especiales cuando el nivel del agua se encuentra cerca o sobre la superficie del suelo.

Es muy importante conservar el grado de saturación de la muestra y de la probeta cuando es manejada en el laboratorio, ya que los resultados se afectan por cambios en el grado de saturación.

En los suelos arcillosos, se encuentra que los parámetros dinámicos son aún más sensibles a estos cambios que los estáticos (Zeevaert, 1997).

Labrada la probeta se coloca en un equipo que admita probetas del orden de $7.5 \mathrm{~cm}$ de diámetro y entre 15 a $20 \mathrm{~cm}$ de altura. Enseguida, se procede a confinarla con el esfuerzo octaédrico efectivo que tenía en campo a la profundidad de su obtención, la cual se estima para el campo por medio de la siguiente fórmula básica:

$$
\sigma c / \sigma z=(1+v) / 3(1-v)
$$

en donde $\sigma z$ es el esfuerzo de sobrecarga en el campo y la relación de Poisson para determinado suelo. (Aproximadamente en arenas $(0.25)$ y arcillas $(0.40)$.

Una vez confinada la probeta se le aplica una distorsión angular previamente programada con el brazo B, Fig. 1 y de inmediato se permite que la probeta vibre libremente. La energía potencial que origina la distorsión inicial se 
convierte en energía cinética de vibración, la cual se disipa gradualmente por amortiguamiento y se registra para efectuar luego el cálculo de las propiedades dinámicas del suelo, Fig. 2, (Zeevaert, 1967 y Van Nostrand, 1983).

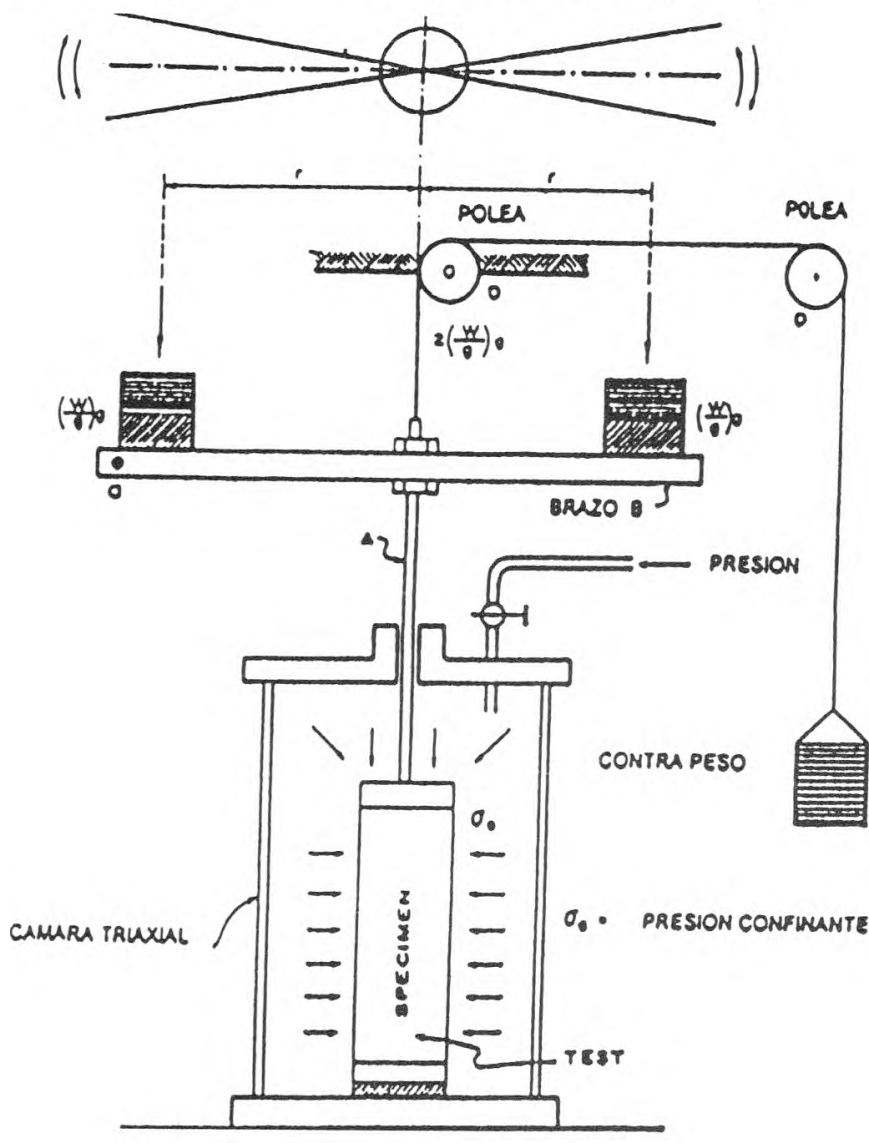

Figura 1. Esquema del péndulo
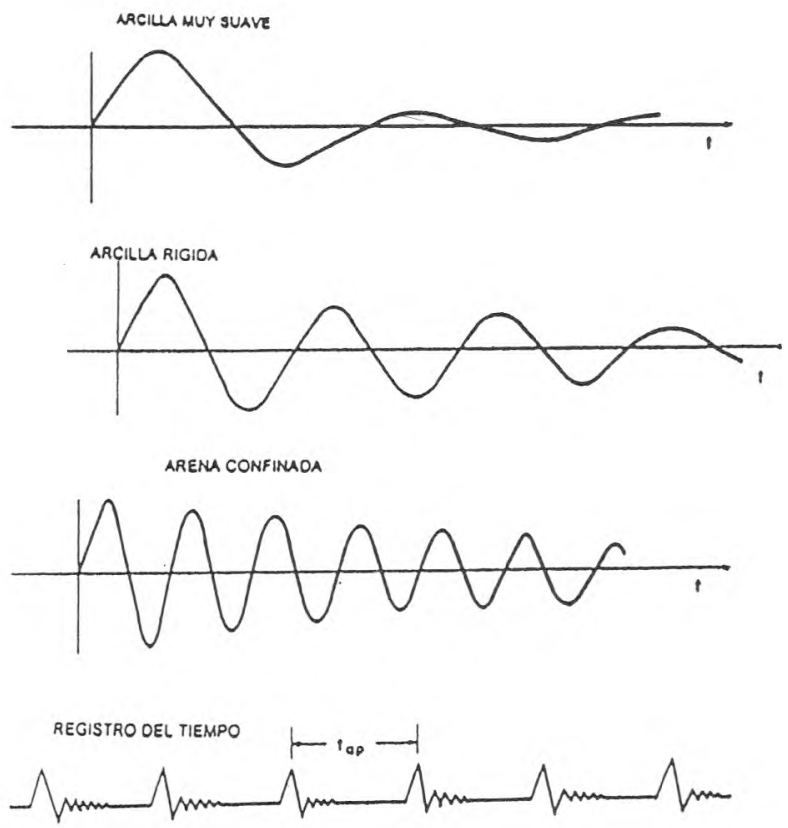

Figura 2. Configuración de registros
El módulo dinámico de rigidez se define a continuación:

$$
\mu=\Delta \mathrm{l} / \Delta \gamma
$$

En donde $\Delta t$ es el incremento de esfuerzo cortante puro que provoca un incremento $\Delta \gamma$ en la distorsión angular, para un determinado estado de esfuerzo octaédrico al que está sujeto el suelo.

El valor de $(\mu)$ no es un parámetro constante, es una función del nivel del esfuerzo octaédrico de la magnitud de la distorsión angular y del grado de saturación (Zeevaert, 1996 y 1967).

En la gráfica se presenta una estadística del módulo dinámico de rigidez para la arcilla limosa típica del subsuelo de la ciudad de México, obtenido de múltiples pruebas e investigaciones por más de dos décadas de práctica profesional del autor, este ejemplo puede servir de motivación para realizar estadísticas de otras regiones afectadas por sismos destructores.

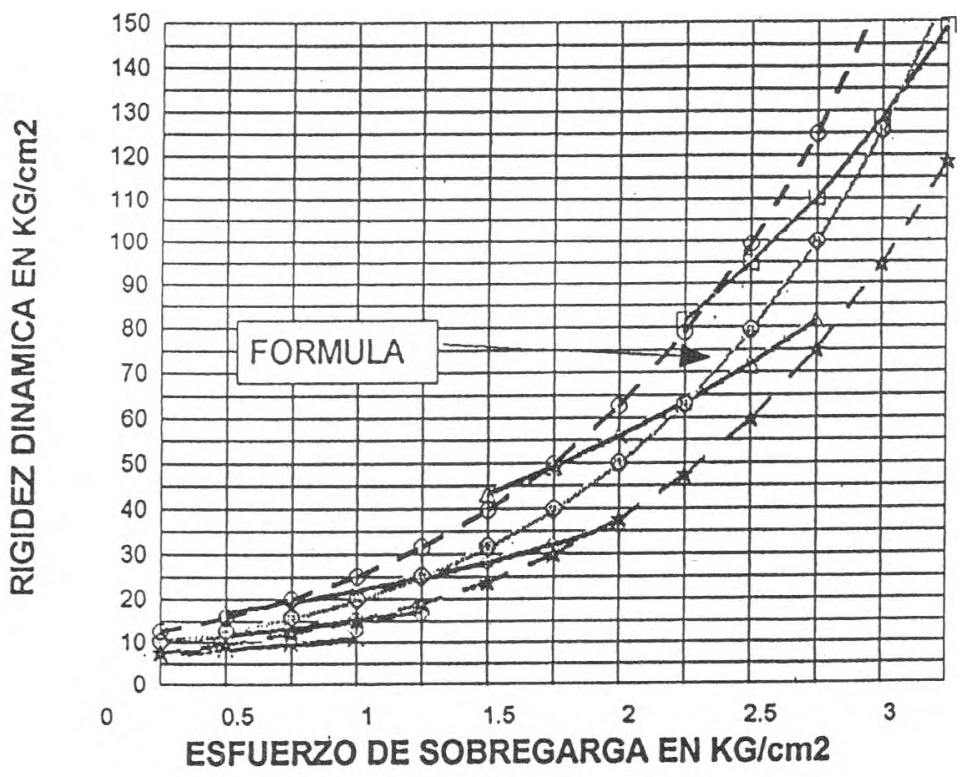

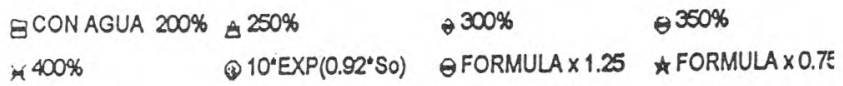

Esfuerzo de sobrecarga en $\mathrm{kg} / \mathrm{cm}^{2}$

De acuerdo con lo anterior, para obtener resultados confiables se debe contar con una muestra inalterada obtenida cuidadosamente, la cual no haya sufrido deterioro en su micro-estructura y mantenga el grado de saturación (\%G) de campo. Por ello, es indispensable que el ingeniero responsable del laboratorio vigile la extracción de las muestras en el campo y ejecute con la técnica que corresponde a cada suelo, asimismo, debe trabajar con el equipo adecuado para su obtención. 
Una vez labrada la probeta, se determina el \%G, el cual resultará algo menor que el de campo, debido a la expansión que sufre el suelo al ser relevado por parte de los esfuerzos efectivos iniciales de campo. Por tanto, es conveniente determinar enseguida, el contenido de agua en el campo, principalmente en muestras cúbicas.

Cuando las muestras se toman con muestreadores especiales se puede calcular de sus características índice, el valor de \%G que corresponde al campo (Zeevaert, 1982).

Una vez montada la probeta en la cámara, se le aplica el esfuerzo octaédrico equivalente al de campo y se observa la deformación volumétrica que sufre el suelo para cersiorarse que se disipó la presión de poro y que la presión aplicada corresponde a esfuerzos efectivos.

Efectuado lo anterior, se procede a realizar la prueba dinámica de torsión libre. Inicialmente, se aplica una distorsión a la probeta con el brazo (B) Fig. 1, dicho giro se programa de acuerdo con el tipo de suelo a investigar (Zeevaert, 1982). Después de aplicar el giro inicial se permite que la probeta responda libremente y así se registre la vibración que muestra por la amplitud amortiguada correspondiente a la disipación de la energía cinética ocasionada por la energía potencial, inducida por la distorsión angular inicial. Los registros que se obtienen son como se muestra en la figura 2 (Íbid).

Del registro del movimeinto amortiguado se obtiene:

a) El período amortiguado.

b) El grado de amortiguamiento.

c) El módulo dinámico de rigidez $(\mu)$.

Las pruebas se llevan a cabo para los diferentes estratos del subsuelo, para conocer el perfil con la profundidad del valor del módulo dinámico de rigidez; sin embargo, se hace hincapié en que una sola prueba de un estrato no es necesariamente representativa del comportamiento dinámico de todo el estrato en cuestión.

Por consiguiente, el ingeniero especialista en estos problemas debe concientizarse de la forma en que se afectan las propiedades cuantitativas de los diferentes suelos por las condiciones ambientales con que se encuentran en la naturaleza y de los cambios que puedan ocurrirles.

Para efectuar correcciones en un mismo tipo de suelo y poder representar el comportamiento dinámico del estrato en su totalidad, se hace necesario efectuar cuando menos, dos pruebas por estrato. Además, es necesario conocer, el perfil con la profundidad de los esfuerzos de sobrecarga, el estado de esfuerzo octaédrico, el grado de saturación y la oquedad del suelo. Para lo anterior, usualmente se determinan estas características a cada $20.30 \mathrm{~cm}$ de profundidad.

Es bien sabido que el especialista de esta disciplina conoce que las propiedades mecánicas y dinámicas cuantitativas dependen de las propiedades físicas del suelo, es decir, un suelo arcillo-limoso, tiene propiedades diferentes a un suelo limo-arcilloso, más aún, si este contiene arena y diferente grado de saturación.

Hay que tener presente que la naturaleza provee al suelo con diferentes características y propiedades físicas de acuerdo con su origen y ambiente, es decir, donde se sedimenta o se forma por la acción ambiental (Van Impe, 1977).

Las leyes fenomenológicas que rigen el comportamiento dinámico para un determinado grado de saturación son las siguientes (Íbid):

En los suelos donde dominan las arcillas:

$$
\mu=(\mu o)=\operatorname{EXP}(\eta c \sigma c) \text { en } \mathrm{kg} / \mathrm{cm}^{2}
$$

En los suelos donde dominan los limos y arenas:

$$
\mu=(\mathrm{Cs})(\sigma c) \eta_{s}, \text { en } \mathrm{kg} / \mathrm{cm}^{2}
$$

En donde:

Ho Módulo para confinamiento octaédrico nulo en $\mathrm{kg} / \mathrm{cm}^{2}$. $\eta$ c Para las arcillas en $\mathrm{kg} / \mathrm{cm}^{2}$.

$\eta$ s Para las arenas y limos (suelos no cohesivos).

Cs Para las arenas y limos (suelos no cohesivos).

бc Confinamiento octaédrico de campo en $\mathrm{kg} / \mathrm{cm}^{2}$.

EXP Base de logaritmos neperianos e $=2.71828$.

La magnitud de los valores de $\eta \mathbf{c}, \mu o, \eta \mathrm{s}$ y $\mathrm{Cs}$ dependen de las características físicas del suelo; sin embargo, no varían en forma muy significativa para un mismo suelo, ni para un estrato en particular. Por tanto, se pueden establecer relaciones correctivas con las ecuaciones 2 y 3 . En el caso de las arcillas es importante corregir el valor de la cohesión por el probable cambio en el grado de saturación con que se efectuó la prueba con respecto al determinado en el campo.

Para dar una idea del orden de magnitud de los valores antes mencionados, se tiene para una arena de compasidad media bien graduada $\mathrm{Cs}=250$ y $\eta \mathrm{s}=0.6 \mathrm{y}$ en el caso de la arcilla solidificada de la ciudad de México para contenidos de agua entre $200 \%$ a $400 \%$, se tienen valores del orden de $\eta \mathrm{c}=0.72 \mathrm{~cm}^{2} / \mathrm{kg}$ y $\mu O=10 \mathrm{~kg} / \mathrm{cm}^{2}$ con variación de $\pm 25 \%$. En la gráfica se presenta una estadística del módulo dinámico de rigidez (Zeevaert, 1996). 
DOI: http://dx.doi.org/10.22201/fi.25940732e.2000.01n4.018

\section{Conclusiones}

El módulo dinámico de rigidez debe investigarse racionalmente para su aplicación en la práctica profesional de todas las universidades y centros de investigación, esto con el propósito de avanzar en la tecnología de la sismo. geodinámica de los suelos, ya que tiene fundamental aplicación en los problemas de estabilidad que se representan en las zonas con acciones sísmicas importantes como es la ciudad de México, la costa del Pacífico de la república mexicana y las regiones sísmicas de otros países.

Hoy en día, a la vista del tercer milenio no se puede evadir la investigación para obtener los conocimientos racionales de la sismo-geodinámica del subsuelo, que representen en forma fidedigna su comportamiento dinámico en la naturaleza.

Es obvio que cada zona sísmica debe desarrollar su propia tecnología de acuerdo con el ambiente dominante y con las características de los suelos en dicha región.

Así también, los laboratorios académicos y profesionales de investigación, deben actualizar sus conocimientos y el equipo de laboratorio para lograr competir y rebatir la tecnología internacional; asimismo, renovar los métodos empíricos nacionales y extranjeros que todavía se utilizan.

Las referencias que se mencionan a continuación, corresponden a libros, conferencias e investigaciones que son resultado de estudios realizados por el autor, los cuales han podido ser liberados ya que al ser aplicados durante años a la práctica profesional, han proporcionado resultados satisfactorios.

Sin embargo, se requiere que los ingenieros estudiosos continúen con las investigaciones en esta disciplina para mejor comprensión y avance de la tecnología de esta rama de la ingeniería.

La literatura es extensa en este tema y el estudioso puede analizarla e interpretarla, asimismo estudiar su veracidad en relación con la realidad que acontece en la naturaleza y en los ambientes para los cuales fue realizada. Es importante conocer los preceptos que indica el profesor Karl Terzaghi, pionero de mecánica de suelos y de problemas en campo (Zeevaert, 1977).

"Entender los fenómenos de la naturaleza es una tarea dificil y de tiempo que el ingeniero cientifico tiene que descubrir sin desesperarse..."

Leonardo Zeevaert (1984)

\section{Referencias}

Harding B.O. y Denevich V.P. (1978). Shear Modulus and Damping in Soils. Proceedings SM7, Journal American Society of Civil Engineers.

Nadai A. (1950). Teory of Flow. Mc-Graw-Hill.

Parton I.M. (1972). Site Response to Earthquakes. Phd. Tesis de la Universidad de Aukland, Nueva Zelanda.

Taylor P.W. y Parton I.M. (1973). Dynamic Testing of Soils, en: Proceedings of the 8th. International Conference of Soil Mechanics and Found, Vol. 1, p. 435, Engineering Moscow. USSR.

Van-Impe W. (1977). De Vrije Wringings Proef Een Belangrijke Bijdrage Tut De Studie Van Het Fundirungen Under Anuloed Van Grongtringen. Tijschrift Van Openbare Werke. Bruselas, Bélgica.

Van-Nostrand R. (1983). Foundation Engineering for Dificult Subsiol Conditions, Segunda Edición.

Zeevaert-Wiechers L. (1997). Conceptos de aplicación para el campo de la resistencia del suelo al esfuerzo cortante. Universidad Nacional Autónoma de Puebla. México.

Zeevaert-Wiechers L. (1997). Riesgo de mal comportamiento de la superficie del suelo y cimentaciones. Conferencia magistral para 40 años de vida de la Sociedad Mexicana de Mecánica de Suelos. México.

Zeevaert-Wiechers L. (1988). Sismo-Geodinámica de la superficie del suelo. Sociedad Mexicana de Mecánica de Suelos. México.

Zeevaert-Wiechers L. (1993). Diseño sismo-geodinámico de cimentaciones. Guayaquil, Ecuador. Reproducido por la División de Estudios de Posgrado de la Facultad de Ingeniería, UNAM. México.

Zeevaert-Wiechers L. (1996). Estabilidad sismo-geodinámica de las cimentaciones. Segundo Seminario Internacional de Geotécnia. Universidad EAFIT de Medellín, Colombia.

Zeevaert-Wiechers L. (1982). Seismic Pore Water Pressure Analysis Confronted with Field Measurements in Fine Sand. Soils and Foundations Engineering. Japanese Society of Soil Mechanics and Foundations, Vol. 23, No. 14.

Zeevaert-Wiechers L. (1996). The Seismic-Geodynamics in the Design of Foundations in Difficult Subsoil Conditions. 3rd. International Symposium on Environmental Geotechnology, San Diego, California.

Zeevaert-Wiechers L. (1979). Deep Foundation Design Problems Related with Ground Surface Subsidence, en: Preceedings Sixth Southeast Asian Conference on Soil Engineering. Vol. 2, 71-110.

Zeevaert-Wiechers L. (1995). Estabilidad sismo-geodinámica de las cimentaciones. Simposio internacional a 10 años del sismo de 1985, SMMS, SMIS, y Centro Nacional de Prevención de Desastres CENAPRED. 
Zeevaert-Wiechers L. (1996). Seismic Soil-Structure Interaction of Foundations with Large Diameter Piles. Proceedings of the Pakistán Academy of Sciences. Vol. 33,1-2.

Zeevaert-Wiechers L. (1). Las anclas en suelos granulares cementados con arcilla. Sociedad Mexicana de Mecánica Suelos. México.

Zeevaert-Wiechers L. (1982). Teoría y práctica del péndulo de torsión libre. División de Estudios de Posgrado de la Facultad de Ingeniería, UNAM. México.

Zeevaert-Wiechers L. (1967). Free Vibration Torsion Test to Determine the Shear Modulus of Elasticity of Soils.
Proceedings 3rd. Panamerican Conference. Soil Mechanics and Foundation Engineering Paper. Sociedad Venezolana de Mecánica de Suelos.

Zeevaert-Wiechers L. (2). Modulus and Damping of Soils by the Resonant Column Method. STP. ASTM.

Zeevaert-Wiechers L. (1977). Seismo Soil Dynamics Response of the Ground Surface and Building Foundations in Mexico City, Earthquake, september 1985.23rd. Terzaghi Lecture, Convención en la Sociedad Americana de Ingenieros Civiles, Anaheim, California.

\section{Semblanza del autor}

Leonardo Zeevaert-Wiechers. Obtuvo el título como ingeniero civil en 1939 en la Escuela Nacional de Ingenieros de la UNAM. Estudio posgrado en el Instituto Tecnológico de Massachusetts donde residió el grado de maestro en ingeniería en 1940. En 1943 inició una estrecha colaboración con el Dr. Karl Terzaghi en una investigación acerca de la estabilidad de las cortinas de corazón hidráulico construidas en México a principios de siglo, esto lo llevó en 1947 a trabajar en problemas especiales de mecánica de suelos en la universidad de Illinois, donde obtuvo el grado de doctor ( $\mathrm{Ph}$. D) en 1949. Ha recibido numerosos reconocimientos, entre ellos: la medalla de oro profesional otorgada por el Instituto Americano de Arquitectos, diploma a la innovación tecnológica, la elección de profesor emérito y miembro de la Academia Nacional de Ingeniería de EUA, entre otros. El buen comportamiento de obras de ingeniería que ha diseñado, así como la cimentación y estructura de la Torre Latinoamericana, en donde introdujo el concepto de flexibilidad controlada en edificios altos, le ha valido para su reconocimiento a nivel internacional. Ha escrito 160 artículos, una gran cantidad de libros; y ha presentado ponencias relacionadas con mecánica de suelos, ingeniería de cimentaciones y sísmica de cimentaciones. 